\title{
Féeries
}

Études sur le conte merveilleux, XVII ${ }^{\mathrm{e}} \mathrm{XIX}{ }^{\mathrm{e}}$ siècle

$10 \mid 2013$

Conte et croyance

\section{Les contes orientaux de Jean Potocki}

The Potocki's Oriental Tales

\section{Dominique Triaire}

\section{OpenEdition}

Journals

Édition électronique

URL : http://journals.openedition.org/feeries/889

DOI : $10.4000 /$ feeries.889

ISSN : 1957-7753

\section{Éditeur}

UGA Éditions/Université Grenoble Alpes

\section{Édition imprimée}

Date de publication : 20 septembre 2013

Pagination : 169-179

ISBN : 978-2-84310-253-0

ISSN : 1766-2842

\section{Référence électronique}

Dominique Triaire, «Les contes orientaux de Jean Potocki », Féeries [En ligne], 10 | 2013, mis en ligne le 20 mars 2015, consulté le 08 septembre 2020. URL : http://journals.openedition.org/feeries/889 ; DOI : https://doi.org/10.4000/feeries.889

\section{(c) Féeries}




\section{LES CONTES ORIENTAUX DE JEAN POTOCKI}

A PART DE LA FICTION occupe peu de place dans l'œuvre de Jean Potocki. En écartant sa modeste production théâtrale, il semble que le Manuscrit trouvé à Saragosse ait aspiré toutes les ressources de son imaginaire. Non qu'il se détourne du récit : tant dans ses relations de voyages que dans ses travaux historiques, il y recourt volontiers, même s'il s'en abstrait progressivement en y insérant des "fragments» extérieurs, puis en le réduisant à une longue théorie de dates et de noms disposés en colonnes ${ }^{\mathrm{I}}$.

Il convient donc d'accorder la plus grande attention à neuf contes orientaux qu'il écrivit entre 1785 et I79I (c'est-à-dire dans la période qui précède la mise en chantier du grand roman), d'autant qu'en soumettant l'un de ces contes à Johann Herder et, derrière lui, à Goethe ${ }^{2}$, il souligne assez l'intérêt qu'il leur portait.

Les cinq premiers contes sont réunis dans le même ensemble ${ }^{3}$ :

- le conte sans titre de la Lettre VI (I, 23-25)

- Le Procès de Draco (I, 25-26)

- Le Songe de Tomrut (I, 27-28)

- Le Voyage de Feirouz (I, 29-30)

- Abdul et Zeila (I, 30-35)

Ces cinq contes furent publiés dans la première édition du Voyage en Turquie et en Egypte (Varsovie, I788). La date de leur rédaction est toutefois très antérieure. Ils n'apparaissent pas encore dans le manuscrit le plus ancien ${ }^{4}$,

I. Voir les Fragments historiques et géographiques, Brunsvic, I796; ou les Principes de chronologie, Saint-Pétersbourg, i810.

2. E. Ranocchi, «Une rencontre oubliée : Potocki et Herder à Karlsbad», dans F. Rosset et D. Triaire (éd.), Jean Potocki ou le dédale des Lumières, Montpellier, Presses universitaires de la Méditerranée, 20I0, p. 29-42.

3. Tomaison et pagination entre parenthèses renvoient à J. Potocki, Euvres, Louvain, Peeters, 2004-2006, 6 vol.

4. Lettres du Cte Jean Potocki Chevalier de Malte a sa Mere, Biblioteka Czartoryskich, Cracovie, ms. 735 . 
daté de I784, mais dès l'année suivante, Potocki recommande à Herder, à qui il envoie Feïrouz. Conte, de "le mettre à la suite des quatre autres'». Contrairement donc à ce que l'auteur veut faire croire à ses lecteurs, ces contes n'ont pas été écrits pendant le séjour à Constantinople en juin I784, mais entre l'Italie (pendant sa quarantaine à Venise?), la Pologne et l'Allemagne, quelques mois plus tard.

Ầ ceux-là, il en ajoute un, Hafféz (I, 35-36), dans la seconde édition du Voyage en Turquie et en Égypte (Varsovie, I789), qui fut donc probablement rédigé entre 1788 et 1789 .

En mai 1790 , il donne un «apologue tartare» dans le Journal Hebdomadaire de la Diète (III, 295-296). Parti pour le Maroc, il rapporte à la date du 8 juillet I79I un conte qui lui a été raconté par son "ami Bin-Otman» (I, I09-III). Le neuvième conte enfin, Le Voyage de Hafez (I, I77-I97), est le plus long: il fait suite au Voyage dans l'empire de Maroc (Varsovie, I792); un manuscrit le date de $179 \mathrm{I}^{6}$.

Là s'arrêtent les contes orientaux de Jean Potocki. Les contes ou légendes qu'il donne dans ses ouvrages sur l'histoire des Slaves n'ont rien d'oriental ${ }^{7}$; nous verrons également que les récits du Manuscrit trouvé à Saragosse ne peuvent être considérés comme des contes orientaux. Résumons rapidement ces neuf contes (leur numéro d'ordre servira ensuite à les désigner).

\section{Le conte de la Lettre VI}

Omar, «riche Mollah», convoite la jeune Fatmé, épouse du «beau Cassem ». Il fait appel à «la vieille Emina Hanem, fameuse intriguante». Celle-ci, sous couvert de la religion, laisse une étoffe de grand prix dans le tapis de prière de Cassem. Quand celui-ci la découvre, il l'attribue à un amant de sa femme et la répudie. Sur le conseil d'Emina, Fatmé, accablée, consent à rencontrer Omar qui lui propose le mariage, mais «veut voir sa femme avant de l'épouser». Omar profite, comme on s'en doute, de l'occasion. Emina révèle alors que l'étoffe lui appartient, à Cassem qui comprend son erreur et s'empresse de réparer ses torts.

\section{Le Procès de Draco}

Par sa connaissance de l'islam, Draco, premier drogman de la Porte et chrétien, suscite la jalousie du mufti qui pousse le vizir à demander à Draco

5. E. Ranocchi, art. cité, p. 35.

6. Le Voyage de Hafez, Biblioteka PAN, Kórnik, ms. I48 A.

7. Voir D. Triaire, "Le Moyen Âge de Jean Potocki», dans Revue des Langues Romanes, cxv/I, 20II, p. 37-38 et 45-47. 
"quelle Religion il croit la meilleure». Devinant le piège, celui-ci répond par un «récit» où il montre que les hommes ressemblent à ceux-là qui cherchent un filon d'or dans l'obscurité : chacun croit l'avoir trouvé et pense que «les autres n'ont que le cuivre \& l'étain», mais Dieu seul sait la vérité.

\section{Le Songe de Tomrut}

Le sultan Névescha vient de monter sur le trône et forme «des projets de conquêtes". Le sage Tomrut lui raconte un songe où un paisible ruisseau se transforme en "un torrent redoutable»; dans la plaine, il s'élargit et disparait, ne laissant "que la mémoire [de ses] ravages». Le sultan écouta le philosophe, mais «le lendemain il fit déclarer la guerre au Sultan de la Perse».

\section{Le Voyage de Feirouz}

Feirouz rentre de pèlerinage aux «Villes Saintes». À la demande de ses enfants, il leur raconte son voyage, mais c'est un conte à leur intention où les pays traversés correspondent aux grandes périodes de la vie.

\section{Abdul et Zeila}

Le conte est sensiblement plus long que les précédents et se divise en deux parties. Dans la première, Zeila, femme du sultan, est jetée par son cheval dans un puits et elle tombe dans les bras d'Abdul qui s'y était réfugié. Les jeunes gens s'aiment, mais bientôt Abdul qui est pauvre n'a plus les moyens d'entretenir Zeila et celle-ci le trahit. Dans la seconde partie, Abdul qui a découvert la trahison de Zeila décide de vivre en ermite dans le désert. Renommé pour sa sagesse, le calife lui confie l'éducation de son fils Caïm. Le maître apprend à l'élève à se méfier des femmes. Un jour, Caïm sauve des pillards une caravane et n'accorde aucune attention à une jeune femme "qui lui tendoit les bras»; c'était Azéma, fille du sultan. Elle révèle son amour à son père et celui-ci fait proposer à Caïm la main de sa fille, mais le jeune homme répond qu'il ne se mariera «que lorsqu'Abdul lui en aura donné l'exemple». Zeila se rend alors auprès d'Abdul, avoue sa faute, les anciens amants se reconnaissent, se pardonnent : Azéma et Caïm peuvent se marier.

\section{Hafféz}

Ce conte et le suivant sont les deux plus courts. Le sage Hafféz «a su rénoncer » à l'amour, aux richesses, à la vengeance, mais «la belle Nourmahal» renverse en un instant toutes les fortes résolutions du sage. 


\section{L'«apologue tartare» du Journal Hebdomadaire de la Diète}

Les Turcs furent délivrés de la servitude au VI ${ }^{\mathrm{e}}$ siècle, mais cent ans plus tôt (et le conte commence ici), un roi voisin leur avait proposé de les libérer à condition qu'ils restent toujours ses amis. Pour s'en assurer, ils les avaient plongés dans le sommeil afin de "connoître leurs véritables sentimens". Découvrant alors que leurs promesses n'étaient rien moins que sûres, le roi préféra les abandonner à leur état.

\section{Le conte de Bin-Otman}

Un habitant de Fez sort de sa maison et, se retournant, croit voir un homme y entrer. Furieux, il revient sur ses pas, frappe à la porte : sa femme apparaît à la fenêtre, s'avoue coupable et demande sa mère. Par une ruse portant sur l'objet de la faute, celle-ci détourne la culpabilité de sa fille et permet à l'amant de s'échapper. Le mari en vient à douter de ce qu'il a vu et finit par s'apaiser.

\section{Le Voyage de Hafez}

$\mathrm{Ce}$ « récit oriental» compte trente et un chapitres et peut se diviser en une introduction (chapitres I-II), trois parties : Hatem Tä et les ingrats (III-XII), considérations politiques et économiques (XIII-XXIV), Medgenoun ou la fin de l'amour (XXV-XXX), et une conclusion (XXXI).

Hafez est un jeune homme qui rencontre sur la route de Bagdad le derviche Bektasch. Ils décident de visiter Hatem Taï, «le meilleur, \& le plus généreux de tous les musulmans", mais à mesure qu'ils s'approchent de sa tribu, les éloges se refroidissent et le vertueux Hatem leur révèle ses "chagrins domestiques». Ils prennent ensuite le chemin d'El-Catif qui s'est libéré de ses oppresseurs, mais le bonheur attendu s'est déjà dissipé. Puis ils se dirigent vers Basra où ils constatent les méfaits dus aux obstacles qui entravent le commerce. Dernière étape : Schiraz où ils font connaissance avec Medgenoun qui suivait l'exemple du héros homonyme avant que ne s'épuise son amour pour Leïlé. Quand Hafez et Bektasch doivent se séparer, celui-ci remet en question l'utilité des voyages.

Quelle définition donner aux textes que nous étudions ici? L'auteur n'en fournit aucune, mais les noms par lesquels il les désigne méritent examen. Le plus fréquent est récit; nous trouvons aussi conte (conteur, conter), oriental, apologue, aventure, fable. Le récit est la «narration d'une aventure»; l'apologue est une «instruction morale qu'on tire de quelque fable inventée exprès" (Dictionnaire de Trévoux, I704). La convergence des noms utilisés 
par Potocki se manifeste clairement dans les deux citations. L'aventure appelle un effet de surprise, elle est unique (au singulier, singulière). Le caractère fictif est fermement souligné - ce qui n'empêche pas la vraisemblance, comme je le montrerai un peu plus loin. La portée morale est explicite, mais pour quel but Potocki donne-t-il une portée morale à ses contes? Cette portée morale n'est-elle qu'un prétexte? n'est-elle qu'une convention? Ou n'est-elle là que pour concentrer les attaques de l'auteur? Questions auxquelles il faudra venir.

Autre information fournie par l'auteur : l'origine du texte.

Pour le conte $\mathrm{I}:$ «Un conteur de profession»

Pour les contes 2 à $7:$ : je» (Potocki)

Pour le conte 8 : «Bin-Otman»

Pour le conte 9 : Jean Potocki (sur la page de titre du volume)

On a vu que les six premiers contes avaient été rédigés bien après le voyage en Turquie et qu'ils étaient à coup sûr sortis de la plume de Potocki; aucun doute non plus pour les contes 7 et 9. Le cas du conte 8 est un peu particulier : Sidi Mohammed ben Othmân, ambassadeur du Maroc à la cour d'Espagne, a bien existé et Jean Potocki l'a rencontré lors de son passage à Madrid en I79I. Il est donc parfaitement possible que le diplomate soit l'auteur du conte, mais Potocki reconnaît lui-même que la transmission a été orale; la version écrite lui revient donc. Assumer un récit, c’est lui donner une force de vérité, compenser son caractère fictif. Dans le cas présent, c'est aussi garantir son "orientalité» en soulignant l'intention esthétique de l'auteur («les agréments de l'élocution orientale», «rendre avec exactitude leurs figures \& leurs expressions", "conserver à mes figures leur physionomie orientale»). Le conte I, attribué à «Un conteur de profession", sert en quelque sorte d'étalon pour les suivants : pur artifice où se révèle le goût de Potocki pour le pastiche ou la fausse référence.

Outre la géographie orientale (Constantinople, l'Indostan, Samarcande, Gazna, le Zendérout, Fez, etc.), toujours soigneusement indiquée, la définition du conte peut encore être affinée. Il est court; le conte 9 fait moins de vingt pages et les autres n'excèdent pas six pages - ce qui contraint l'auteur à une rigoureuse économie : peu de descriptions (balancées par la précision géographique), peu d'introspections (le caractère des personnages se devine à travers leurs propos), unité d'action, sauf pour le conte 5 - même le conte 9 qui se découpe en épisodes successifs obéit à une composition linéaire. Autre caractéristique du conte : la narration à la troisième personne à la différence du Manuscrit trouvé à Saragosse. La disposition du conte est donc l'inverse de celle du roman : ici, la narration à la première personne est introduite par un auteur de troisième personne, Alphonse excepté : 
«Pascheco poussa un horrible hurlement et commença en ces termes : [...] Je suis né à Cordoue» (IV, I, 52-53). Dans les contes, l'introduction est à la première personne : "en voici une que j'entendis raconter", "J'ai cherché de même à rendre». Dans le Manuscrit trouvé à Saragosse, l'énoncé se veut authentique (autobiographique) alors que l'appareil de l'énonciation (le voyage d'Alphonse et au-delà le manuscrit espagnol retrouvé) est fabuleux. Dans le conte, l'effort d'authenticité porte non sur l'énoncé (même si elle doit être vraisemblable, l'histoire est fictive), mais sur l'énonciation : l'auteur ne se dissimule pas.

Le conte oriental de Potocki présente une autre caractéristique : il ne s'y trouve nulle trace de merveilleux ou de surnaturel. Quand il repose sur une action (contes I et 8), elle se développe avec la plus exacte vraisemblance; quand il se construit autour d'un récit interne (contes 2, 3, 4, 6, 7), celui-ci, loin d'être un divertissement hétérogène, est étroitement lié aux conditions de son apparition : ainsi Feirouz qui rentre de voyage raconte-t-il un voyage, Tomrut, assis au bord d'un fleuve, y trouve-t-il l'argument de son récit, et par un jeu à multiples détentes, le conte 6 renvoie à un ouvrage de Joseph de Guignes qui renvoie lui-même à la situation de la Pologne. Ce fort ancrage de l'énoncé dans l'énonciation ne se retrouvera pas dans le Manuscrit trouvé à Saragosse.

La rhétorique enfin participe à la définition du conte. Dès qu'il affirme son autorité, dans l'introduction du conte 2 , Potocki la relie à un style à la «maniere» des Orientaux qui se caractérise dans la phrase par un recours à la comparaison ou à la métaphore : «l'hypocrite est comme le roseau [...]» (I, 24), "la faveur des Princes est une toile qu'un Peintre a remplie [...]» (I, 33), etc. Ces figures restent toutefois peu fréquentes dans les huit premiers contes, à l'exception du conte 6 qui repose sur la contradiction entre les principes moraux, illustrés par des figures, et le comportement du héros. Dans le dernier conte, la fréquence augmente (chapitres xii, xiv, xv, xix, xxv, xxx, xxxi). Avec une dizaine de comparaisons ou métaphores, Potocki a voulu garder mesure, éviter un excès rhétorique qui aurait donné à ses contes une teinte baroque. En revanche, chaque figure est plus développée que dans l'usage occidental et donne lieu au moins à une proposition complète : «ceux qui travaillent, gardent le silence, \& c'est ainsi que dans nos jardins vous n'entendez ni l'araignée ni le vers à soye, tandis que vous êtes étourdi par les cigales \& les hannetons» (I, I85); la figure est un objet travaillé, précis. La distance entre le comparant et le terme comparé doit être importante et provoque un effet de surprise (d'autant plus fort que la figure est rare) : hypocrite / roseau, faveur / peinture, ceux qui travaillent / araignée et vers à soie, etc. Cette distance sémantique des deux termes rend 
parfois la comparaison étrange, voire obscure, renforçant ainsi le caractère exotique du conte :

Seigneur (lui répondit Bektasch) je ne suis point entièrement de votre avis; il est possible que le nom de Hatem si révéré ici, le soit beaucoup moins dans sa tribu, \& encore moins dans sa famille. Lorsque l'on voit de loin les piramides de Firaoun, elles paroîssent toucher au ciel; mais lorsqu'on s'en approche, l'on voit qu'elles ne sont habitées que par des jackals \& des chauve-souris. (I, I79)

L'attachement des Turcs aux animaux, aux arbres, à la nature, signalé dans la Lettre X (I, 38), oriente le choix du comparant : roseau, eau, fange, araignée, cigales, abeilles, etc. Très souvent, la figure ferme un récit (conte 3) ou un chapitre (conte 9), unissant à la comparaison la chute; elle se substitue ainsi à la sentence (qui termine les contes I, 4, 5), marquée par une grande banalité : «ne méprisons point des gens dont le métier est de ne faire que des heureux» (I, 25). La figure est donc soulignée par l'originalité des termes qui la constitue, son emplacement, le contraste qu'elle offre avec la sentence; elle ruine ainsi toute portée morale du conte, l'orientant vers (et le réduisant à) cette chute non moins frappante qu'inattendue ${ }^{8}$.

La fin du conte 9 donne lieu à trois prosopopées : la porte (ch. XXVII), la fontaine (ch. XXVIII), les myrtes (ch. XXIX). À la vérité, ce ne sont pas de véritables prosopopées puisque ce n'est pas la porte qui parle; une main y a inscrit : "Je suis la porte qui conduit" (I, I93). Pas de merveilleux donc, comme nous l'avons déjà dit, et resserrement entre le terme figuré (un locuteur) et l'objet réel. Ces prosopopées illustrent l'histoire de Medgenoun et Leïlé, mais il ne s'agit que de deux jeunes gens qui, emportés par leur amour, s'identifient aux héros du poème, mais rapprocher le modèle idéal de sa copie réelle (au lieu de les éloigner comme dans les figures précédentes) mène à l'échec : l'amour du nouveau Medgenoun et de la nouvelle Leïlé s'est épuisé'.

Il est un autre recours à la rhétorique opéré par Potocki : le narrateur enchâsse un récit allégorique, censé éclairer (plutôt qu’illustrer) son propos moral, philosophique, politique, etc. Le récit est supérieur en taille à la figure : il s'étend du paragraphe (la goutte d'eau, I, 33) au conte presque entier (conte 4). Sur l'ensemble des contes, il se trouve six récits : la mine (I, 26), le ruisseau (I, 28), le voyage (I, 29), la goutte d'eau (I, 33), les noms des voyageurs (I, I77), le clou et la vis (I, I9I). Tous les récits enchâssés ne sont pas rhétoriques; n’entrent pas dans cette catégorie le récit de vie (I, 23 :

8. Comme dans le proverbe, forme théâtrale pratiquée par Potocki (III, 85).

9. Même épuisement entre les deux cousines dans le Manuscrit trouvé à Saragosse (première journée). 
les pèlerinages d'Emina), la lettre ou le chant de Zeila (I, 3I-32). Comme pour la figure, Potocki veille à ne pas tomber dans l'excès, ce qui donne plus de relief au procédé - l'enchâssement sera beaucoup plus fréquent dans le Manuscrit trouvé à Saragosse. Comme pour la figure, l'écart entre le récit allégorique et le propos qu'il éclaire doit frapper : la mine, l'obscurité, la fourmi pour décrire... les religions et montrer explicitement qu'aucune ne peut prétendre être plus vraie qu'une autre, implicitement qu'elles aveuglent et asservissent ceux qui les croient. Parfois l'écart est tel que non seulement le rapport entre récit et propos finit par être insaisissable, mais encore que le récit lui-même devient abscons : quel rapport entre l'ermite intimidé par la présence du calife et la goutte d'eau devenue perle? dans le clou et la vis, il semble que Potocki se joue de son lecteur puisque le récit se développe sur le thème des "raisonnemens faux \& absurdes». Inversement, le récit peut s'ancrer dans le réel, le métamorphoser, puis délivrer enfin et presque accessoirement le propos : des noms des voyageurs, gravés sur le mur d'un caravansérail, à une variante orientalisante du carpe diem - ou plutôt noctem. La surprise ne vient donc pas de l'opposition entre naturel et surnaturel (ou merveilleux) comme un conte oriental le laisserait attendre, mais du décalage entre le texte et l'image : soit elle ne représente pas le texte, soit elle manque de netteté, soit elle transforme le texte, censé dire le réel. Le tout sans excès, sans la violence du fantastique, mais en insinuant, par les jeux de contraste entre les termes de la comparaison, une impression d'étrangeté, de malaise (ai-je mal lu?) face à une parole qui n'est pas tout à fait ce qu'elle devrait être.

Les contes orientaux de Jean Potocki ne peuvent toutefois se réduire à une forme, aussi séduisante soit-elle, ou même à un stratagème finement imaginé - comme celui de la vieille Emina Hanem ou de la mère de l'épouse infidèle au conte 8 . Ce serait oublier la grande cohérence qu'ils présentent dans l'analyse extrêmement négative de la condition humaine. Hommes et femmes (et surtout les femmes) ne sont guidés que par l'intérêt (Emina Hanem, les femmes de Hatem Taï), la concupiscence (Omar, conte I), la jalousie (Chek-al-Islam, conte 2), la soif de pouvoir (Névescha, conte 3). Pour arriver à leurs fins, ils ne reculent devant aucune traitrise comme Zeila (conte 5) qui tente de supprimer son amant quand il a épuisé pour elle ses ressources aussi bien pécuniaires que sexuelles. Les victimes ne sont pas épargnées : nulle plainte de Fatmé qui a dû subir les assauts d'Omar (une satisfaction secrète? "malgré les richesses du Mollah, elle se crut heureuse de retrouver son époux", I, 25); Abdul, ayant découvert que Zeila voulait sa mort, se réfugie dans le désert et devient une espèce de dévot aigri, mais 
quand il la revoit, il cède au sentiment, donnant l'image d'un être faible et velléitaire. Le mari du conte 8 , doublement trompé par sa femme et la mère de celle-ci, doute moins de leurs paroles trompeuses que de ses propres yeux. Celui qui fait le bien n'est récompensé que par la plus noire ingratitude comme le roi de Touran (conte 7) ou Hatem Taï (conte 9).

Les trois quarts de la population, ceux qui travaillent, ne portent aucun intérêt à la politique, mais le «quatrième quart, composé de gens oisifs, qui ont le tems de gouverner les autres, [...] sont eux-mêmes gouvernés par trois ou quatre beaux parleurs» (I, I84). Le conte 9 montre comment la politique donne lieu à la stupidité telle qu'elle se déploie dans le protectionnisme des maîtres de l'Irak et du Hedgiage (ch. XVI), à la rapacité qui affame les plus faibles (ch. XVII), à la malhonnêteté enfin (ch. XVIII). Loin de jouer un rôle modérateur, la religion sert à tromper ou à assouvir les passions coupables (conte I) et ne fait qu'attiser la haine (conte 2). Et quand une révolution renverse l'ordre (ou plutôt le désordre) des choses, quelques mois suffisent :

non seulement pour modérer la joye d'un peuple, mais même pour le dégoûter de la plus heureuse révolution; qui d'ailleurs ne tient jamais tout le bonheur qu'elle promettoit, mais au contraire amène seulement une manière d'être différente à la vérité, mais toujours également mêlée de bien \& de mal. (I, I83-I84)

La sagesse est inefficace : le sultan Névescha (conte 3) néglige les conseils du philosophe Tomrut et part en guerre. Hafféz, qui s'est entouré d'inscriptions qui célèbrent sa sagesse, ne peut supporter d'être joué par la «belle Nourmahal» et la tue ainsi que ses prétendants avant de se suicider (conte 6). L'amour le plus pur, quand il n'est pas dénaturé par l'infidélité ou l'ingratitude, ne dure jamais bien longtemps et il n'a pas fallu quatre ans pour que l'amour de Medgenoun et de Leïlé qui «n'éprouvoit d'obstacle que dans son excès» (I, 194) finisse, amenant la séparation des deux amants.

De ce sombre tableau, une leçon peut-elle être tirée? Doit-on se laisser gagner par le découragement? Il est certain que les contes sont empreints d'un profond scepticisme : peu de foi en la nature humaine, aucune assurance dans le savoir. Faut-il se résoudre au sort de Hafféz (qui sera celui de Potocki)? L'Orient ouvre toutefois quelques pistes. Bektasch délivre à son jeune disciple une morale qui peut l'aider à vivre :

je me suis beaucoup occupé d'études abstraites, \& je voyage aujourd'hui autant pour m'en distraire que pour ajouter, la connoissance des hommes, aux connoissances que j'ai puisées dans les livres; seigneur (lui répondit Bektasch.) Vous verrez partout plus de mal que de bien; mais vous ne verrez nulle part le mal, sans mélange d'un peu de bien, \& cela doit suffire au sage pour le consoler de la vie. (I, I78) 
Trois points doivent ici être soulignés : l'importance du voyage dont Potocki sera toujours convaincu et qui vient en complément au livre. L'étude est ce qui compense chez Medgenoun le vide laissé par l'amour (I, I95); c'est aussi l'occupation principale de Jean Potocki quand il écrit ses contes : "Nous faisons une vray vie d'hermites. $\mathrm{M}^{\mathrm{r}}$ Jean a donné dans le gout de grandes etudes C'est du grec et de la cronologie toute la journée ${ }^{\mathrm{I0}} »$ écrit sa belle-mère, la princesse maréchale Lubomirska, le I3 août 1785 de Bagni di Lucca. On retrouve enfin, dans ces lignes du conte 9, cette philosophie de la mesure, de l'équilibre, que Potocki développait en 1790 dans son Essay d'aphorismes sur la liberté (III, 3IO) et qui préférait à des réformes brutales, dangereuses et incertaines, des améliorations modestes et prudentes.

Tout n'est donc pas perdu et si l'expérience politique de Potocki dans les années de la grande diète de Pologne et lors de son séjour parisien de I790-I79I lui inspire le ton désabusé du conte 9, il y a toujours «un peu de bien». Une forme de bonheur simple (rousseauiste?) peut même être recherchée; elle reparaît à quelques reprises : le bonheur "est par-tout; mais le Voyageur égaré ne le cherche ni dans l'éclair qui l'éblouit, ni dans le feu follet qui glisse sur la fange; s'il le trouve, c'est dans le caillou qu'il fouloit à ses pieds» $(\mathrm{I}, 30)$. Pour autant que la figure se laisse saisir, le bonheur n'est donc ni dans l'éclat passager de la fortune, ni dans le succès éphémère, mais dans les satisfactions humbles et solides. Le bonheur se trouve en chacun et ne s'attache qu'au seul présent : "C'est de lui qu'il faut jouir", chante Zeila (I, 3I). Feïrouz enseigne à son fils : «si tu ne cherches le bonheur que dans toi-même, mon but sera rempli \& je n'aurai rien à desirer» (I, 30). Outre la gloire fugace ( le feu follet»), il faut éviter un bonheur qui s’appuierait sur autrui; ce serait faire fonds sur du sable. Il est cependant une relation qui peut apporter du réconfort : non celle de la femme, pas même celle de l'ami, mais celle du frère (I, I95-196). Sans doute est-ce encore là l'expérience qui affleure : on sait l'affection de Jean Potocki pour son frère Séverin. Les conseils pour trouver le bonheur sont donc bien courts, mais n'oublions pas Hafféz : les conseils du sage eux-mêmes n'échappent pas au scepticisme, à l'incertitude, voire à l'erreur, et les multiplier multiplie aussi les risques d'échec.

Le dernier conte oriental date de I79I ; le premier état du Manuscrit trouvé à Saragosse date de 1794, mais la forme avancée du texte prouve que la mise en chantier remonte assurément à plusieurs mois, peut-être plusieurs

Io. AGAD, Varsovie, Archives publiques des Potocki 293, f. 2.

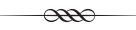




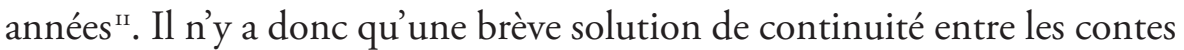
et le roman, seuls récits de fiction (retrouvés à ce jour) de l'œuvre de Jean Potocki. J'ai relevé les différences entre les deux ensembles, mais il existe aussi des analogies. Dans la version de $1794 \mathrm{du}$ Manuscrit, la couleur orientale est portée par l'histoire du juif errant qui trouve ici sa plus grande extension - elle sera sensiblement réduite dans la version de I804, supprimée dans la version de I8ıo. Y reparaît également la fourberie sous les traits de Hillel, beau-père de Hiskias ${ }^{12}$. Mais l'histoire de Velasquez prend, par rapport aux contes, une orientation nouvelle : la méchanceté humaine s'efface pour céder la place aux caprices de la fortune dont sera victime le père du géomètre. En revanche, le moyen de les supporter, d'atteindre sinon au bonheur, au moins à une certaine sérénité, se trouve dans l'étude, comme le recommandait Medgenoun (I, I95). Il semble donc que le scepticisme cruel, teinté d'indifférence, enrobé dans un style orientalisant, exprimé par des récits brefs et légers, s'estompe dans les premières journées du Manuscrit trouvé à Saragosse. Il pouvait être l'effet de l'influence parisienne ou de la cour de Stanislas Auguste; le deuxième partage de la Pologne, les temps sombres de la Révolution française durent inciter le jeune homme à un peu plus de profondeur.

II. Voir F. Rosset et D. Triaire, «La première version du Manuscrit trouvé à Saragosse», dans É. Klene (éd.), Jean Potocki à nouveau, Amsterdam/New York, Rodopi, 20IO, p. 323-43I.

I2. Ibid., p. 358 et suiv. 